\title{
Early Methanogenic Colonisation in the Faeces of Meishan and Yorkshire Piglets as Determined by Pyrosequencing Analysis
}

\author{
Yong Su, ${ }^{1}$ Gaorui Bian, ${ }^{1}$ Zhigang Zhu, ${ }^{1}$ Hauke Smidt, ${ }^{2}$ and Weiyun $\mathrm{Zhu}^{1}$ \\ ${ }^{1}$ Laboratory of Gastrointestinal Microbiology, College of Animal Science and Technology, Nanjing Agricultural University, \\ Nanjing 210095, China \\ ${ }^{2}$ Laboratory of Microbiology, Wageningen University, Dreijenplein 10, Wageningen $6703 \mathrm{HB}$, The Netherlands
}

Correspondence should be addressed to Weiyun Zhu; zhuweiyun@njau.edu.cn

Received 21 September 2013; Accepted 18 December 2013; Published 6 January 2014

Academic Editor: Masaaki Morikawa

Copyright (C) 2014 Yong Su et al. This is an open access article distributed under the Creative Commons Attribution License, which permits unrestricted use, distribution, and reproduction in any medium, provided the original work is properly cited.

\begin{abstract}
Gut methanogenic archaea of monogastric animals are considered to be related to energy metabolism and adipose deposition of the host; however, information on their development in young piglets is limited. Thus, to investigate early methanogenic colonisation in the faeces of Meishan and Yorkshire piglets, faecal samples were collected from piglets at 1, 3, 7, and 14 days after birth and used to analyse the methanogenic community with $16 \mathrm{~S}$ rRNA gene pyrosequencing. Results showed that the diversity of the methanogenic community in the faeces of neonatal piglets decreased from one to 14 days of age, as the total methanogen populations increased. The age of piglets, but not the breed, significantly affected the diversity of the methanogenic community which was dominated by the genus Methanobrevibacter. From the ages of one to 14 days, the abundance of $M$. smithii-related operational taxonomic units (OTUs) increased significantly, while the abundances of $M$. thaueri- and M. millerae-related OTUs decreased significantly. The substitution of M. smithii for M. thaueri/M. millerae was faster in Yorkshire piglets than in Meishan piglets. These results suggest that the early establishment of microbiota in neonatal piglets is accompanied by dramatic changes in the methanogenic community, and that the changes vary among pigs of different genotypes.
\end{abstract}

\section{Introduction}

Methanogenic archaea exist widely in the gastrointestinal (GI) tract of many vertebrates and invertebrates [1-3]. Methanogens can use hydrogen and other compounds such as formate, methanol, and acetate, as electron donors for the production of methane. Methane formation not only contributes to global warming as a greenhouse gas, but it also represents an energy loss for the animal [4]. Recently, methanogenic archaea in the gut of monogastric animals, including humans, have been studied intensively because gut methanogens are considered to be related to energy metabolism and adipose deposition of the host [5]. In addition, methane produced by methanogens might play an important role in the pathogenesis of several intestinal disorders, including colon cancer, inflammatory bowel disease, irritable bowel syndrome, and diverticulosis [6]. Thus far, only limited reports on gut methanogens in pigs have been available. Based on archaeal $16 \mathrm{~S}$ ribosomal RNA (rRNA) gene clone library analysis, methanogens belonging to the genus Methanobrevibacter were found to be predominant in pig faeces $[7,8]$.

The GI microbiota of newborn animals play a fundamentally important role in the development of intestinal function and the innate immune system [9-12]. The infant gut ecosystem undergoes a dramatic transition from an essentially sterile state to extremely dense colonisation, ending with the establishment of an adult-like microbial community $[13,14]$. In contrast to the gut microbiota of adult animals, the microbiota of neonates are more variable and less stable over time. The fragile ecological system is not only a disease risk to the newborn gut, but it can also have a long-term effect on it's later life health [15-17]. Comparing the gut bacterial communities of neonatal animals and humans which have been intensively studied, information on gut methanogenic communities of neonatal monogastric animals such as pigs is still limited. 
The Meishan and Yorkshire breeds are typical obese and lean pigs, respectively; thus, their energy metabolism might be distinctive. It has been found that obese Meishan pigs harbour relatively higher numbers of Firmicutes and lower numbers of Bacteroidetes compared to lean breeds [18]. Moreover, a recent study showed that lean Landrace pigs harboured a greater diversity of methanogens and a higher number of methanogen $m c r A$ gene copies than the obese Erhualian pigs [8]. However, it is not clear whether the different composition of gut methanogens in the various pig breeds is related to the early methanogenic colonisation of newborn piglets. Therefore, the aim of this study was to investigate the development of methanogenic archaea in the faeces of newborn Meishan and Yorkshire piglets by using high throughput pyrosequencing analysis of PCR-amplified 16S rRNA genes.

\section{Materials and Methods}

2.1. Collection of Faecal Samples. This study was approved by the Nanjing Agricultural University Animal Care and Use Committee. All of the Meishan and Yorkshire pigs were raised on a commercial farm in Jiangsu province, China. Candidate sows with a similar expected delivery date were chosen from both breeds and injected intramuscularly with cloprostenol (0.2 mg per sow) at 10:00 am on day 113 of pregnancy to ensure homochronous deliveries. Four vaginally delivered litters of piglets (each litter with 10-12 piglets) for each pig breed which delivered homochronously within two hours were finally used in this study. The same diets were formulated for Meishan and Yorkshire sows according to nutrient requirements of the National Research Council. Fresh faeces were collected from the piglets at 1, 3, 7 and 14 days of age and immediately stored at $-28^{\circ} \mathrm{C}$ for further molecular analysis.

2.2. DNA Extraction and PCR Amplification. Total genomic DNA was isolated from the faecal samples using a commercially available stool DNA extraction kit, according to the instructions of the manufacturer (QIAamp DNA Stool Mini Kit: Qiagen, Hilden, Germany). The concentration of the extracted DNA was determined using a NanoDrop 1000 spectrophotometer (Thermo Scientific Inc., Wilmington, DE, USA).

To analyse the taxonomic composition of the methanogenic community, Archaea-specific primers (Arch344F 5'ACG GGG YGC AGC AGG CGC GA-3' and Arch915R 5' GTG CTC CCC CGC CAA TTC CT- $3^{\prime}$ ) targeting the V3-V6 region of the $16 \mathrm{~S}$ rRNA gene were chosen for the amplification and subsequent pyrosequencing of the PCR products $[19,20]$. The PCRs were carried out in triplicate in $50 \mu \mathrm{L}$ reactions with $10 \mu \mathrm{L} 5$-fold reaction buffer, $50 \mathrm{ng}$ of DNA, $0.4 \mathrm{mM}$ of each primer, 0.5 U Pfu polymerase (TransStart-FastPfu DNA Polymerase, TransGen Biotech), and $2.5 \mathrm{mM}$ dNTPs. The amplification program consisted of an initial denaturation step at $95^{\circ} \mathrm{C}$ for $2 \mathrm{~min}$. This was followed by 30 cycles, where one cycle consisted of $95^{\circ} \mathrm{C}$ for $30 \mathrm{~s}$ (denaturation), $58^{\circ} \mathrm{C}$ for $90 \mathrm{~s}$ (annealing), $72^{\circ} \mathrm{C}$ for $30 \mathrm{~s}$ (extension), and a final extension of $72^{\circ} \mathrm{C}$ for $5 \mathrm{~min}$. PCR products were visualised on agarose gels ( $2 \%$ in TBE buffer) containing ethidium bromide, and purified with a DNA gel extraction kit (Axygen, China).

2.3. Pyrosequencing and Bioinformatics. Prior to sequencing, the DNA concentration of each PCR product was determined using a Quant-iT PicoGreen double-stranded DNA assay (Invitrogen, Germany) and was quality-controlled on an Agilent 2100 Bioanalyzer (Agilent, USA). Amplicon pyrosequencing was performed from the A end using a 454/Roche A sequencing primer kit on a Roche Genome Sequencer GSFLX Titanium platform at Majorbio Bio-Pharm Technology Co., Ltd., Shanghai, China.

PCR-amplified fragments were blunted and tagged on both ends with ligation adaptors that contained a unique $10 \mathrm{bp}$ sequence (sample specific barcode sequence) and a short 4-nucleotide sequence (TCAG) called sequencing key, which were recognised by the system software and the priming sequences. All pyrosequencing reads were binned according to barcode and primer sequences. The resulting sequences were further screened and filtered for quality. Sequences that were shorter than 200 bp in length, contained ambiguous characters, contained over two mismatches to the primers, or contained mononucleotide repeats of over six nt were removed. To assess bacterial diversity among samples in a comparable manner, a randomly selected, 2564sequence (the lowest number of sequences in the 32 samples) subset from each sample was aligned using the "align.seqs" command and compared with the SILVA archaeal database (SILVA version 108). The aligned sequences were further trimmed and the redundant reads were eliminated using successively the "screen.seqs", "filter.seqs," and "unique.seqs" commands. The "chimera.slayer" command was used to determine chimeric sequences. The "dist.seqs" command was performed, and unique sequences were clustered into operational taxonomic units (OTUs) defined by $97 \%$ similarity [21] using CD-HIT-OUT program [22]. We also calculated the coverage percentage using Good's method [23], abundance-based coverage estimator (ACE), bias-corrected Chao richness estimator, and the Shannon and Simpson diversity indices. A heat map was generated using custom Perl scripts. All the analyses were performed using the MOTHUR program (http://www.mothur.org/) [24]. Principal coordinate analysis (PCoA) was conducted based on the weighted UniFrac distance [25].

2.4. Phylogenetic Analysis. Sequences of OTUs with an abundance higher than $0.1 \%$ with total reads were derived and used for construction of a phylogenetic tree. Homology searches of the GenBank DNA database were further performed with a BLAST search. Sequences of OTUs-related species were retrieved from the GenBank database. Multiple sequence alignments were performed using ClustalX1.81 [26]. Phylogenetic analysis was performed with the MEGA 3.1 software package. An unrooted phylogenetic tree was constructed using the neighbour-joining method [27].

2.5. Real-Time PCR Quantification of Methanogenic Archaea. Quantitative PCR was performed on an Applied Biosystems 
7300 Real-Time PCR System (ABI) using SYBR Green as the fluorescent dye. The reaction mixture $(25 \mu \mathrm{L})$ consisted of $12.5 \mu \mathrm{L}$ of IQ SYBR Green Supermix (Bio-Rad), $0.2 \mu \mathrm{M}$ of each primer set, and $5 \mu \mathrm{L}$ of the template DNA. The amount of DNA in each sample was determined in triplicate, and the mean values were calculated. Archaea-specific primers Arch $344 \mathrm{f} \mathrm{[19])} \mathrm{and} \mathrm{Arch806} \mathrm{[28]} \mathrm{were} \mathrm{used} \mathrm{to} \mathrm{quantify} \mathrm{the}$ $16 \mathrm{~S}$ rRNA gene of archaeal methanogens under the following conditions: an initial DNA denaturation step at $95^{\circ} \mathrm{C}$ for $10 \mathrm{~min}$, followed by 40 cycles of denaturation at $95^{\circ} \mathrm{C}$ for $15 \mathrm{~s}$, and primer annealing and extension at $60^{\circ} \mathrm{C}$ for $1 \mathrm{~min}$. DNA from cells of a pure culture of $M$. smithii was used as the standard. Results are expressed as the numbers of $16 \mathrm{~S}$ rRNA gene copies per gram of faeces.

2.6. Statistical Analysis. The effects of pig breed and age on the composition of the methanogenic archaeal community were tested for significance using a two-way analysis of variance (ANOVA) program in the Statistical Package for the Social Sciences (SPSS17.0). Significant differences were declared when $P<0.05$.

\section{Results}

3.1. Metrics of Pyrosequencing Analysis. Across all 32 samples, 132138 quality-trimmed sequences from a total of 165338 reads were classified as Archaea. The average length of the quality-trimmed sequences was $483 \mathrm{bp}$. The rarefaction curves generated by MOTHUR plotting the number of reads against the number of OTUs indicated that using 2564 reads per sample (the minimum number of sequences passing all quality control measures across all samples) for the final analysis was adequate as the curves tended to approach the saturation plateau (Figure 1).

3.2. Diversity. Coverage, number of OTUs, and statistical estimates of species richness for each group at a genetic distance of $3 \%$ are presented in Table 1 . The age of the piglets significantly affected the diversity indices (Shannon and Simpson) and richness estimators (ACE and Chao) of faecal methanogenic archaeal community $(P<0.05)$; there was no significant difference between the pig breeds. In both breeds, the piglets harboured a higher diversity of faecal methanogens at 1 and 3 days of age than at 7 and 14 days $(P<0.05)$.

3.3. Taxonomic Composition. Across all reads, $99.91 \%$ were identified as class Methanobacteria, while Thermoplasmatales composed the remaining $0.09 \%$. Within class Methanobacteria, family Methanobacteriaceae was predominant, represented by genera Methanobrevibacter and Methanosphaera. A very high abundance of genus Methanobrevibacter (95.01\%$100 \%$ ) was found in all samples; thus, further analysis was performed at the species (OTU) level.

Clustered heat map analysis based on the archaeal community profiles at the OTU level showed that most samples taken from the piglets at 1 and 3 days of age were grouped together and separated from the samples taken at 7 and 14

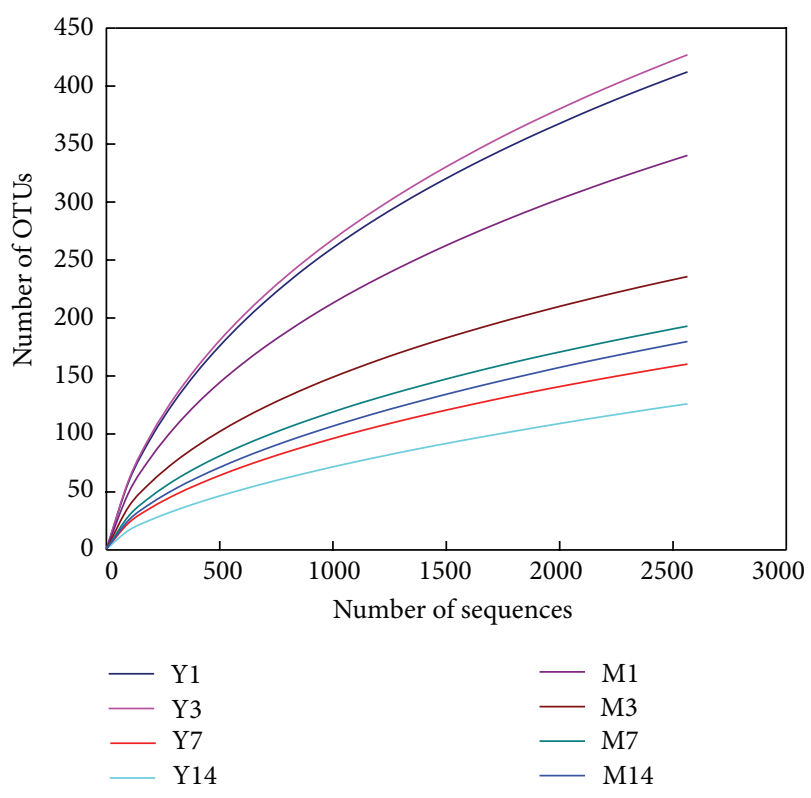

FIGURE 1: Rarefaction curves comparing the number of reads with the number of phylotypes (OTUs) found in the 16S rRNA gene libraries from faecal methanogens of Meishan and Yorkshire piglets. M: Meishan piglets; Y: Yorkshire piglets; 1, 3, 7, and 14 represent the ages of $1,3,7$, and 14 days.

days (Figure 2). In addition, PCoA analysis also showed that the first principal coordinate (P1), which explains $44.0 \%$ of the variation, separated the archaeal communities of most of the piglets at 7 and 14 days from the samples of younger animals (Figure 3 ).

Sequences of predominant OTUs with a relative abundance higher than $0.5 \%$ of total reads and reference sequences of Methanobrevibacter spp. were used for construction of the phylogenetic tree. All of the OTUs were closely related to genus Methanobrevibacter (Figure 4). Most of the OTUs were divided into two clusters-M. smithii/M. woesei cluster and $M$. thaueri/M. millerae cluster. OTUs $1,2,3,4,13,20$, and 28 were most closely related to M. smithii, whereas OTUs $5,6,7,8,9,14,19,29$, and 30 were most closely related to $M$. thaueri. OTUs with a relative abundance higher than $0.1 \%$ of total reads were further used for a significance test of relative abundance among different groups (Table 2). OTUs significantly affected by pig breed or age were mainly related to M. smithii, M. thaueri, and M. millerae $(P<$ $0.05)$. Pig breed significantly affected the relative abundances of M. smithii- and M. thaueri-related OTUs, and pig age significantly affected the relative abundances of predominant M. smithii-, M. thaueri-, and M. millerae-related OTUs $(P<0.05)$ (Table 3). Interestingly, it was observed that the substitution of M. smithii for M. thaueri/M. millerae occurred faster in Yorkshire piglets than in Meishan piglets. Sequences most closely related to $M$. smithii became predominant at the age of 7 days in Yorkshire piglets, whereas this species dominated in the Meishan piglets only from day 14. In both breeds, from day 1 to day 14 , the relative abundance of $M$. smithii-related OTUs increased significantly in the faeces, 


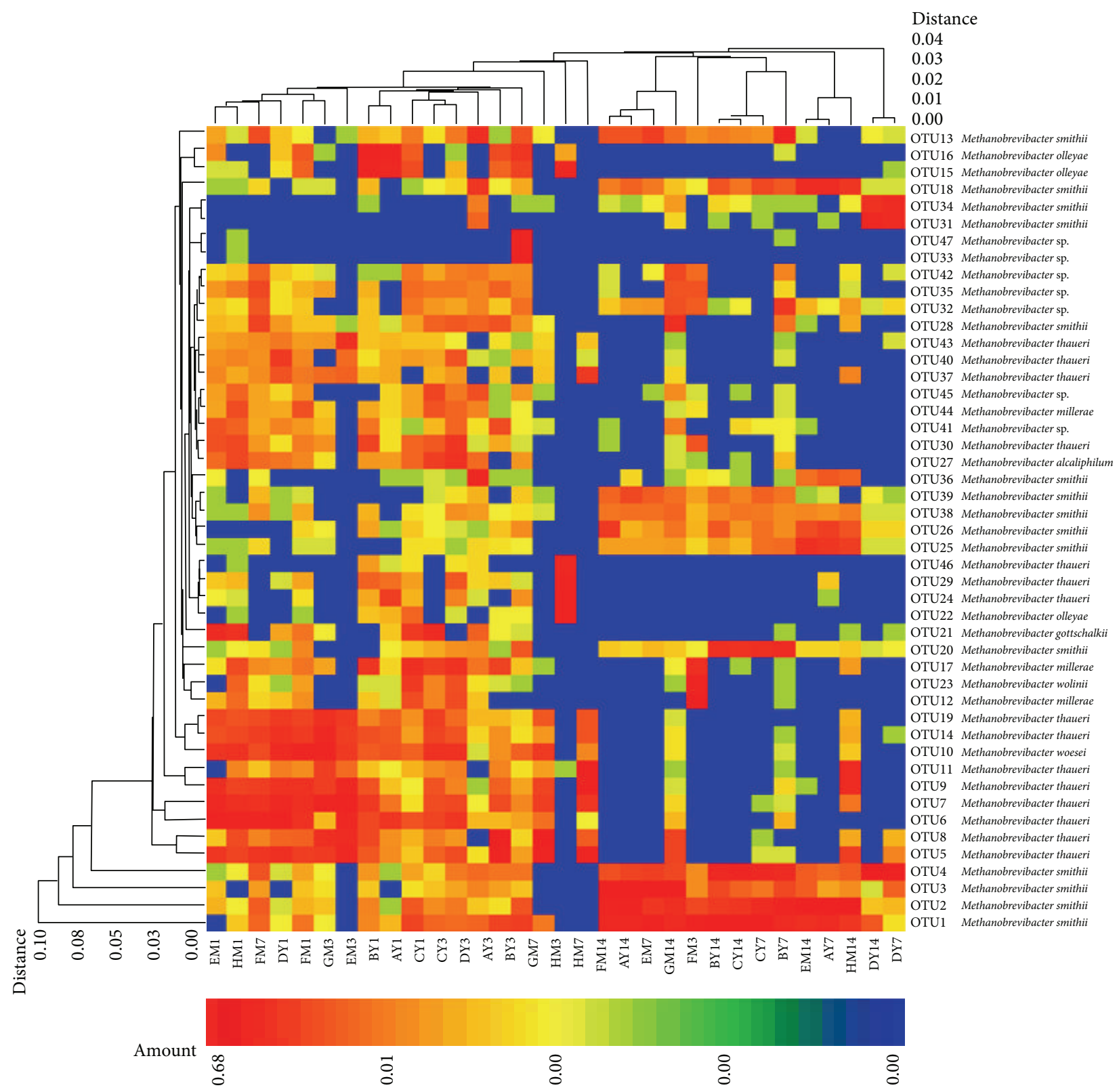

FIgURE 2: Double dendrogram showing the distribution of methanogens in the faeces of Meishan and Yorkshire piglets (OTU level). The relationship among samples was determined using Bray distance and the complete clustering method. A total of 47 OTUs with an abundance higher than $0.3 \%$ within total methanogens were selected for the analysis. The heatmap plot depicts the relative abundance of each OTU (variables clustering on the $y$-axis) within each sample ( $x$-axis clustering). The relative values for the OTUs are depicted by colour intensity in the legend at the top of the figure. Clusters based on the distance of all samples along the $x$-axis and the different OTUs along the $y$-axis are indicated at the top and left of the figure, respectively. A-D: litter numbers of Yorkshire piglets; E-H: litter numbers of Meishan piglets.

while the relative abundances of $M$. thaueri- and $M$. milleraerelated OTUs decreased significantly $(P<0.05)$.

\subsection{Quantification of $16 S$ rRNA Gene Copies of Methanogenic} Archaea. The absolute numbers of the $16 \mathrm{~S}$ rRNA gene copies of methanogenic archaea in the faeces of the piglets were determined with real-time PCR assays (Figure 5). Pig breed did not affect faecal methanogen populations; however, in both breeds, significant differences were found among samples taken from the piglets at different ages. The numbers of faecal methanogen $16 \mathrm{~S}$ rRNA gene copies found in the piglets at 7 and 14 days were significantly higher than those found at 1 and 3 days $(P<0.05)$.

\section{Discussion}

Since Ley et al. found that gut microbiota were associated with the energy metabolism of the host, the role of gut microbiota in the metabolism of the host has received more attention [29]. While numerous studies have focused on two 
TABLE 1: Phylotype coverage and diversity estimation of the 16S rRNA gene libraries from faecal methanogens of Meishan and Yorkshire piglets ${ }^{1}$.

\begin{tabular}{|c|c|c|c|c|c|c|c|}
\hline Breed & Age (d) & OTUs & $\mathrm{ACE}$ & Chao & Shannon & Simpson & Coverage \\
\hline \multirow{4}{*}{ Meishan } & 1 & 340.3 & 697.1 & 564.2 & 4.263 & 0.045 & 0.938 \\
\hline & 3 & 235.8 & 484.0 & 398.3 & 3.551 & 0.085 & 0.958 \\
\hline & 7 & 193.0 & 470.9 & 343.1 & 2.797 & 0.207 & 0.963 \\
\hline & 14 & 179.8 & 502.4 & 353.9 & 2.505 & 0.279 & 0.963 \\
\hline \multirow{4}{*}{ Yorkshire } & 1 & 412.3 & 783.4 & 658.4 & 4.732 & 0.029 & 0.928 \\
\hline & 3 & 427.0 & 791.1 & 689.4 & 4.789 & 0.028 & 0.924 \\
\hline & 7 & 160.3 & 416.8 & 277.8 & 2.311 & 0.326 & 0.968 \\
\hline & 14 & 126.0 & 434.9 & 272.0 & 1.764 & 0.419 & 0.972 \\
\hline \multicolumn{2}{|c|}{$\mathrm{SEM}^{2}$} & 137.7 & 250.95 & 214.0 & 1.297 & 0.168 & 0.023 \\
\hline \multirow{3}{*}{ Effect ( $P$ value $)$} & Breed & 0.184 & 0.409 & 0.307 & 0.664 & 0.185 & 0.236 \\
\hline & Age & 0.000 & 0.048 & 0.001 & 0.000 & 0.000 & 0.001 \\
\hline & Breed $\times$ age & 0.049 & 0.346 & 0.102 & 0.063 & 0.138 & 0.092 \\
\hline
\end{tabular}

${ }^{1}$ The operational taxonomic units (OTUs) were defined with $3 \%$ dissimilarity. The coverage percentages, richness estimators (ACE and Chao), and diversity indices (Shannon and Simpson) were calculated.

${ }^{2}$ SEM: standard error of means, $n=4$.

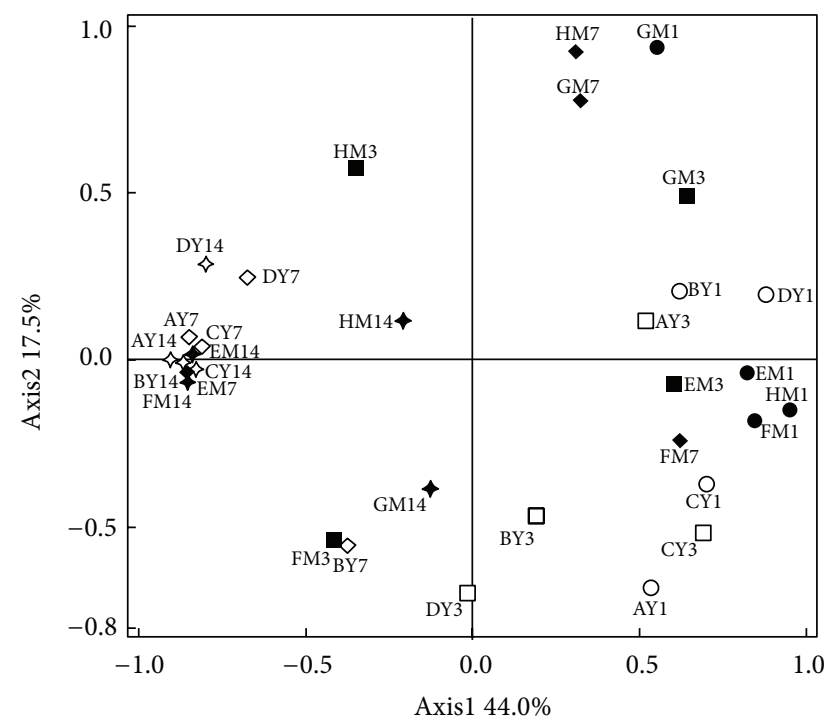

FIgURE 3: Principal coordinates analysis of weighted UniFrac values in the fecal methanogens of Meishan and Yorkshire piglets. Sample identifiers are the same as in Figures 1 and 2.

major bacterial groups (Firmicutes and Bacteroidetes) in the gut of animals, it was shown in a germ-free mouse model that methanogens also play an important role in energy metabolism and adipose deposition [30]. Unlike the potential roles of methanogens in a host's energy metabolism and adipose deposition, the diversity and structure of methanogenic communities in pig gut have not been well understood.

Studies using 16S rRNA gene-based techniques indicate that the predominant species in ruminants belong to the genus Methanobrevibacter [31,32]. Recently, a methanogenic archaeal 16S rRNA gene library of pig faeces was constructed wherein the clones were mainly separated into three clusters-Methanobrevibacter, Methanosphaera, and a group of uncultivated archaea [7]. In our previous study, the genus
Methanobrevibacter was also found to dominate in the faeces of Erhualian and Landrace pigs [8], which is consistent with the result of the present study on Yorkshire and Meishan piglets. However, methanogenic genera besides Methanobrevibacter were seldomly detected in the present study, which suggests that the colonisation of other methanogens might occur later than the colonization of Methanobrevibacter spp.

To date, the diversity of methanogens in the human gut has been thought to be limited to several species among which M. smithii has been regarded as the main methane producer [33]. Furthermore, it has been shown that $M$. smithii concentration was higher in anorexic patients than in a lean population [34]. It was also reported that the gut microbiota from obese individuals were depleted in M. smithii [35]. These results indicate that $M$. smithii might play an important role in host energy metabolism. Similarly, it was observed that the abundance of $M$. smithii-related OTUs within the methanogenic 16S rRNA gene library was significantly higher in lean Landrace pigs than in obese Erhualian pigs [8]. In the present study, we found that $M$. smithii in the faeces of both breeds gradually became predominant during the first two weeks after birth. Furthermore, the dominance of M. smithii in the lean Yorkshire piglets occurred earlier than in obese Meishan piglets, which confirms the previous finding that lean animals and humans may harbour more M. smithii in their gut than obese ones.

The composition and diversity of neonatal microbiota are variable and vulnerable during early life until a stable ecosystem is established. Facultative anaerobes such as Enterobacteriaceae, enterococci, streptococci, and staphylococci, have been reported to be the predominant intestinal microbiota in infants during the first week $[36,37]$, as they can survive in the oxygen-containing gut. As oxygen is comsumed, anaerobic microorganisms, such as Ruminococcus, Bacteroides, and Bifidobacterium, gradually thrive [14, 38, 39]. An increasing diversity in the bacterial community was also observed during the development of gut microbiota in newborn piglets [40]. In contrast to the bacterial community, 


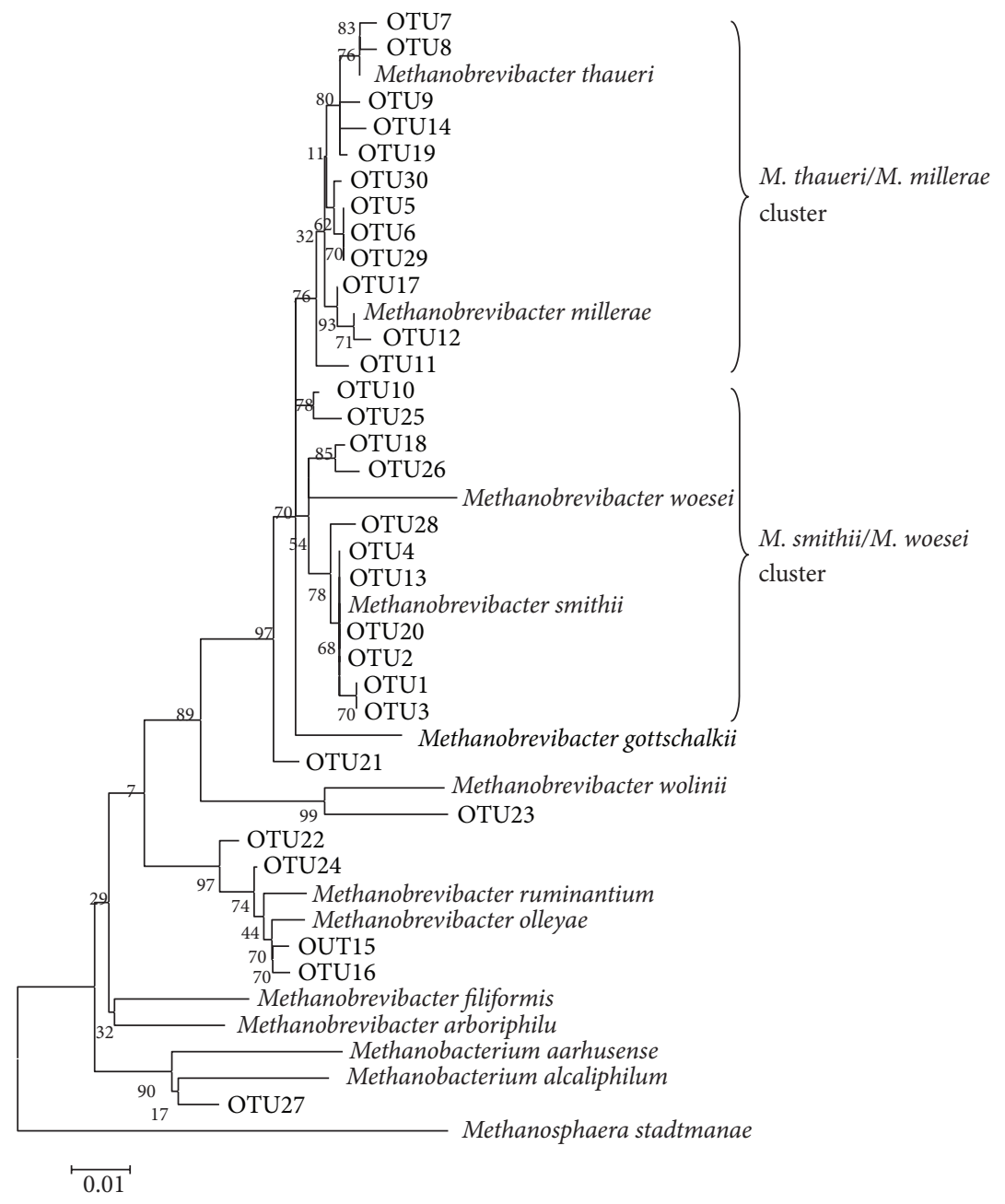

FIGURE 4: Unrooted phylogenetic tree of Methanobrevibacter spp. reference strains and predominant OTUs in the libraries from faecal methanogens of Meishan and Yorkshire piglets. The reference bar indicates $1 \%$ sequence dissimilarity.

we found that the diversity indices of the faecal methanogenic community of Meishan and Yorkshire piglets decreased from 1 day to 14 days, which suggests that the colonisation of gut methanogens in neonatal piglets is the result of mutual selection between the methanogens and the host. This result is also consistent with the previous finding that the diversity of methanogens in the human and monogastric animal gut has been thought to be limited to several species.

In the present study, although the diversity of the methanogen populations decreased with the age of the newborn piglets, the numbers of methanogens increased significantly and reached $10^{9}$ copies of $16 \mathrm{~S}$ rRNA gene per gram of faeces, which is similar to values observed for faeces of grown pigs and suckling piglets before weaning [8]. Combined with the results of diversity and taxonomic composition, this result suggests that a stable methanogenic community is established in the gut of piglets at the age of 14 days.

Interestingly, we found that from day 1 to day 14 , the abundance of $M$. smithii-related OTUs in the faeces increased significantly, while the abundances of M. thaueriand M. millerae-related OTUs decreased significantly. It was found that $M$. thaueri- and M. millerae-related OTUs dominated in the methanogenic 16S rRNA gene library from the faeces of the Meishan and Yorkshire piglets three days after birth. Although these two species could be found in the hindgut of pigs $[8,41]$, M. smithii was the most abundant species in previous studies $[7,8]$. In the gut, M. smithii converts $\mathrm{H}_{2}, \mathrm{CO}_{2}$, and formate into $\mathrm{CH}_{4}$ using carbon as the terminal electron acceptor. In contrast, $M$. thaueri only grows and produces methane from $\mathrm{H}_{2}$ and $\mathrm{CO}_{2}$ and does not grow or produce methane from formate, acetate methanol, trimethylamine, or methanol with $\mathrm{H}_{2}$ [41]. Considering the potential role of $M$. smithii in energy metabolism, this result indicates that the substitution of M. smithii for other Methanobrevibacter spp. might be important to the gut microbial ecosystem. We found that this substitution occurred more quickly in the Yorkshire piglets than in the Meishan piglets. Further studies are still needed to understand whether this difference is related to the different phenotype of these two breeds. 
TABLE 2: Relative abundances of predominant OTUs (percentage) in the 16S rRNA gene libraries from faecal methanogens of Meishan and Yorkshire piglets ${ }^{1}$.

\begin{tabular}{|c|c|c|c|c|c|c|c|c|c|c|c|c|c|}
\hline \multirow{2}{*}{ OTUs } & \multicolumn{4}{|c|}{ Yorkshire } & \multicolumn{4}{|c|}{ Meishan } & \multirow{2}{*}{ SEM $^{2}$} & \multicolumn{3}{|c|}{ Effect ( $P$ value $)$} & \multirow{2}{*}{$\begin{array}{c}\text { Closest } \\
\text { reference strain }\end{array}$} \\
\hline & D1 & D3 & D7 & D14 & D1 & D3 & D7 & D14 & & Breed & Age & Breed $\times$ age & \\
\hline OTU2 & 0.53 & 0.69 & 20.63 & 5.79 & 0.20 & 0.83 & 1.39 & 32.0 & 17.0 & 0.741 & 0.046 & 0.034 & M. smithii \\
\hline OTU6 & 6.89 & 1.20 & 0.09 & 0.00 & 10.16 & 0.68 & 3.41 & 0.08 & 5.07 & 0.293 & 0.001 & 0.679 & M. thaueri \\
\hline OTU7 & 3.19 & 1.31 & 0.03 & 0.00 & 9.87 & 3.92 & 2.97 & 0.26 & 4.50 & 0.024 & 0.012 & 0.392 & M. thaueri \\
\hline OTU10 & 3.64 & 1.57 & 0.02 & 0.00 & 6.14 & 3.22 & 1.60 & 0.10 & 3.23 & 0.157 & 0.011 & 0.860 & M. woesei \\
\hline OTU14 & 2.26 & 0.60 & 0.02 & 0.00 & 2.80 & 2.38 & 1.33 & 0.14 & 1.78 & 0.108 & 0.028 & 0.731 & M. thaueri \\
\hline OTU17 & 2.56 & 2.22 & 0.01 & 0.01 & 0.74 & 2.39 & 0.17 & 0.16 & 1.76 & 0.547 & 0.017 & 0.508 & M. millerae \\
\hline OTU19 & 2.12 & 0.45 & 0.00 & 0.00 & 2.52 & 1.51 & 1.19 & 0.11 & 1.37 & 0.097 & 0.004 & 0.738 & M. thaueri \\
\hline OTU20 & 0.20 & 0.64 & 3.26 & 2.57 & 0.20 & 0.03 & 0.12 & 0.22 & 1.88 & 0.015 & 0.204 & 0.217 & M. smithii \\
\hline OTU21 & 2.89 & 0.35 & 0.02 & 0.00 & 3.23 & 0.03 & 0.00 & 0.01 & 1.98 & 0.997 & 0.002 & 0.984 & M. gottschalkii \\
\hline OTU26 & 0.10 & 0.25 & 1.21 & 0.61 & 0.05 & 0.22 & 0.13 & 2.00 & 0.88 & 0.812 & 0.005 & 0.010 & M. smithii \\
\hline OTU27 & 1.49 & 1.49 & 0.08 & 0.01 & 0.98 & 0.19 & 0.24 & 0.02 & 1.03 & 0.223 & 0.049 & 0.403 & M. alcaliphilum \\
\hline OTU30 & 0.67 & 1.09 & 0.03 & 0.00 & 1.15 & 1.21 & 0.14 & 0.03 & 0.93 & 0.560 & 0.032 & 0.956 & M. thaueri \\
\hline OTU38 & 0.08 & 0.34 & 0.53 & 0.66 & 0.12 & 0.17 & 0.40 & 0.86 & 0.36 & 0.864 & 0.001 & 0.542 & M. smithii \\
\hline OTU39 & 0.03 & 0.19 & 0.55 & 1.00 & 0.03 & 0.12 & 0.41 & 0.52 & 0.53 & 0.324 & 0.026 & 0.756 & M. smithii \\
\hline OTU40 & 1.21 & 0.54 & 0.02 & 0.00 & 0.75 & 0.04 & 0.22 & 0.02 & 0.69 & 0.409 & 0.019 & 0.594 & M. thaueri \\
\hline OTU44 & 0.66 & 0.49 & 0.02 & 0.00 & 1.06 & 0.20 & 0.11 & 0.02 & 0.55 & 0.750 & 0.005 & 0.544 & M. millerae \\
\hline OTU48 & 0.15 & 0.34 & 0.53 & 0.40 & 0.06 & 0.09 & 0.16 & 0.61 & 0.28 & 0.134 & 0.010 & 0.083 & M. millerae \\
\hline OTU52 & 0.56 & 0.05 & 0.01 & 0.00 & 1.00 & 0.40 & 0.19 & 0.02 & 0.53 & 0.139 & 0.012 & 0.796 & M. thaueri \\
\hline OTU63 & 0.42 & 0.20 & 0.01 & 0.00 & 0.80 & 0.17 & 0.09 & 0.01 & 0.30 & 0.077 & 0.000 & 0.088 & M. thaueri \\
\hline OTU69 & 0.59 & 0.20 & 0.02 & 0.00 & 0.51 & 0.06 & 0.20 & 0.01 & 0.27 & 0.875 & 0.000 & 0.299 & M. thaueri \\
\hline OTU71 & 0.26 & 0.31 & 0.00 & 0.00 & 0.68 & 0.14 & 0.14 & 0.01 & 0.31 & 0.285 & 0.005 & 0.146 & M. millerae \\
\hline OTU73 & 0.08 & 0.13 & 0.60 & 0.35 & 0.01 & 0.08 & 0.03 & 0.25 & 0.27 & 0.016 & 0.038 & 0.067 & M. smithii \\
\hline OTU74 & 0.41 & 0.63 & 0.00 & 0.00 & 0.08 & 0.35 & 0.00 & 0.00 & 0.40 & 0.243 & 0.035 & 0.697 & M. olleyae \\
\hline OTU77 & 1.00 & 0.03 & 0.02 & 0.00 & 0.36 & 0.01 & 0.00 & 0.00 & 0.48 & 0.219 & 0.003 & 0.278 & M. millerae \\
\hline OTU78 & 0.38 & 0.15 & 0.02 & 0.00 & 0.51 & 0.09 & 0.20 & 0.02 & 0.23 & 0.263 & 0.000 & 0.487 & M. thaueri \\
\hline OTU82 & 0.28 & 0.14 & 0.01 & 0.00 & 0.33 & 0.07 & 0.40 & 0.05 & 0.21 & 0.075 & 0.010 & 0.047 & M. thaueri \\
\hline OTU85 & 0.25 & 0.13 & 0.00 & 0.00 & 0.39 & 0.35 & 0.10 & 0.02 & 0.26 & 0.175 & 0.047 & 0.862 & M. thaueri \\
\hline OTU86 & 0.17 & 0.16 & 0.02 & 0.00 & 0.44 & 0.16 & 0.29 & 0.00 & 0.20 & 0.024 & 0.010 & 0.153 & M. thaueri \\
\hline OTU88 & 0.33 & 0.04 & 0.00 & 0.00 & 0.49 & 0.20 & 0.12 & 0.00 & 0.25 & 0.143 & 0.002 & 0.845 & M. thaueri \\
\hline OTU91 & 0.67 & 0.07 & 0.00 & 0.00 & 0.33 & 0.00 & 0.04 & 0.01 & 0.35 & 0.399 & 0.006 & 0.572 & M. millerae \\
\hline OTU93 & 0.51 & 0.24 & 0.00 & 0.00 & 0.18 & 0.17 & 0.00 & 0.00 & 0.29 & 0.291 & 0.045 & 0.570 & M. millerae \\
\hline OTU103 & 0.04 & 0.04 & 0.17 & 0.32 & 0.02 & 0.04 & 0.10 & 0.21 & 0.15 & 0.277 & 0.002 & 0.821 & M. smithii \\
\hline OTU104 & 0.26 & 0.10 & 0.00 & 0.00 & 0.24 & 0.22 & 0.10 & 0.00 & 0.17 & 0.338 & 0.010 & 0.706 & M. thaueri \\
\hline OTU107 & 0.16 & 0.06 & 0.00 & 0.00 & 0.47 & 0.05 & 0.14 & 0.00 & 0.18 & 0.007 & 0.000 & 0.017 & M. thaueri \\
\hline OTU109 & 0.16 & 0.38 & 0.03 & 0.00 & 0.20 & 0.04 & 0.03 & 0.02 & 0.19 & 0.217 & 0.038 & 0.073 & M. millerae \\
\hline OTU110 & 0.22 & 0.18 & 0.04 & 0.00 & 0.27 & 0.08 & 0.04 & 0.02 & 0.17 & 0.892 & 0.018 & 0.786 & M. millerae \\
\hline
\end{tabular}

${ }^{1}$ OTUs: the abundances that were significantly affected by pig breed, age, or the interaction between breed and age are shown in this table.

${ }^{2}$ SEM: standard error of means, $n=4$.

The microbiota of newborns mainly travel from the mother's vagina, skin, and faeces $[42,43]$. In addition, diet composition is regarded as the main factor affecting gut microbiota. It was reported that Erhualian (similar breed to Meishan) sows had higher concentrations of milk lactose and fat but lower concentration of milk protein as compared to traditional Western breeds [44], which may be a possible reason for the existence of $M$. thaueri and $M$. millerae in the faeces of newborn piglets in relatively high abundance. Further studies are needed to reveal their potential role in the gut of newborn piglets.

\section{Conclusions}

In conclusion, the present study showed that the methanogenic community in the faeces of Meishan and 
TABle 3: Relative abundances (percentage) of M. smithii-, M. thaueri-, M. millerae-, and M. olleyae-related OTUs in the 16S rRNA gene libraries from faecal methanogens of Meishan and Yorkshire piglets.

\begin{tabular}{|c|c|c|c|c|c|}
\hline \multirow{2}{*}{ Breed } & \multirow{2}{*}{ Age (d) } & \multicolumn{4}{|c|}{ Relative abundance within total methanogens } \\
\hline & & M. smithii-related OTUs & M. thaueri-related OTUs & M. millerae-related OTUs & M. olleyae-related OTU \\
\hline \multirow{4}{*}{ Meishan } & 1 & 1.81 & 61.11 & 18.43 & 1.67 \\
\hline & 3 & 8.13 & 26.79 & 23.68 & 26.25 \\
\hline & 7 & 26.28 & 57.54 & 7.29 & 0.000 \\
\hline & 14 & 78.51 & 7.52 & 6.99 & 0.000 \\
\hline \multirow{4}{*}{ Yorkshire } & 1 & 3.68 & 33.01 & 27.72 & 10.25 \\
\hline & 3 & 16.89 & 18.95 & 24.87 & 5.35 \\
\hline & 7 & 88.41 & 0.79 & 3.74 & 0.13 \\
\hline & 14 & 94.27 & 0.02 & 0.85 & 0.000 \\
\hline \multicolumn{2}{|c|}{ SEM $^{1}$} & 41.67 & 31.78 & 16.06 & 14.53 \\
\hline \multirow{3}{*}{ Effect ( $P$ value $)$} & Breed & 0.003 & 0.010 & 0.969 & 0.521 \\
\hline & Age & 0.000 & 0.020 & 0.009 & 0.081 \\
\hline & Breed $\times$ age & 0.017 & 0.198 & 0.715 & 0.173 \\
\hline
\end{tabular}

${ }^{1}$ SEM: standard error of means, $n=4$.

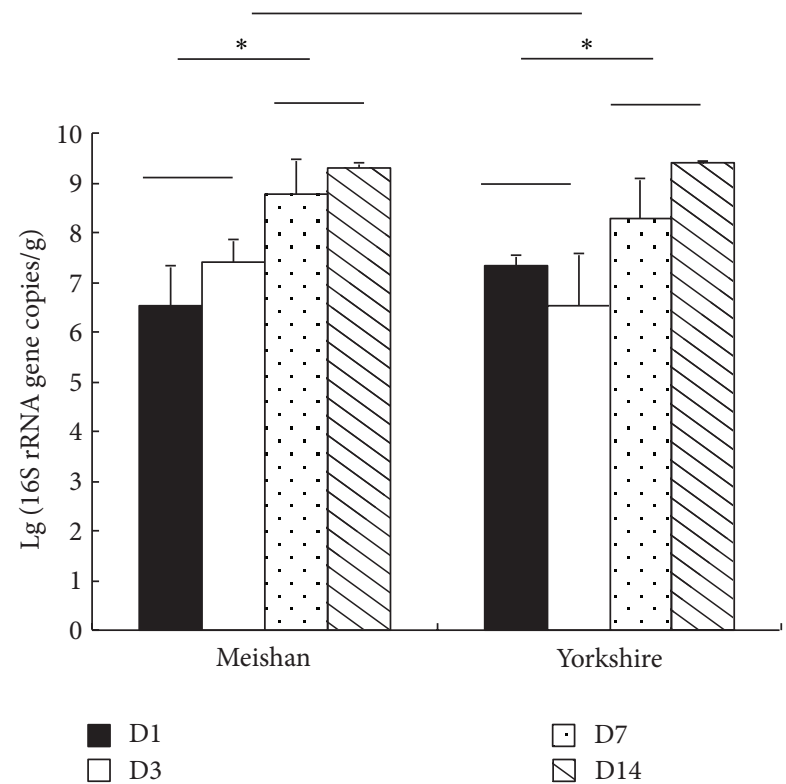

FIGURE 5: Real-time PCR quantification of 16S rRNA gene copies of methanogenic archaea in the faeces of Meishan and Yorkshire piglets. ${ }^{*} P<0.05, n=4$.

Yorkshire neonatal piglets was dominated by members of the genus Methanobrevibacter, represented by M. smithii, $M$. thaueri, and $M$. millerae. The structure of the methanogenic community was significantly affected by the age of piglets; however, pig breed could also affect the substitution of different Methanobrevibacter spp.

\section{Conflict of Interests}

The authors declare that there is no conflict of interests regarding the publication of this paper.

\section{Acknowledgments}

This research has received funding from the National Basic Research Program of China (2012CB124705), the National Natural Science Foundation of China (30810103909), the Fundamental Research Funds for the Central Universities (KYZ201153), the European Community's Seventh Framework Programme (FP7/2007-2013) under the Grant Agreement no. 227549, and the China-EU Science and Technology Cooperation Foundation (1008).

\section{References}

[1] J. H. P. Hackstein and T. A. van Alen, "Fecal methanogens and vertebrate evolution," Evolution, vol. 50, no. 2, pp. 559-572, 1996.

[2] B. Morvan, F. Bonnemoy, G. Fonty, and P. Gouet, "Quantitative determination of $\mathrm{H}_{2}$-utilizing acetogenic and sulfate-reducing bacteria and methanogenic Archaea from digestive tract of different mammals," Current Microbiology, vol. 32, no. 3, pp. 129-133, 1996.

[3] C. Lin and T. L. Miller, "Phylogenetic analysis of Methanobrevibacter isolated from feces of humans and other animals," Archives of Microbiology, vol. 169, no. 5, pp. 397-403, 1998.

[4] K. A. Johnson and D. E. Johnson, "Methane emissions from cattle," Journal of Animal Science, vol. 73, no. 8, pp. 2483-2492, 1995.

[5] B. S. Samuel, E. E. Hansen, J. K. Manchester et al., "Genomic and metabolic adaptations of Methanobrevibacter smithii to the human gut," Proceedings of the National Academy of Sciences of the United States of America, vol. 104, no. 25, pp. 10643-10648, 2007.

[6] D. Roccarina, E. C. Lauritano, M. Gabrielli, F. Franceschi, V. Ojetti, and A. Gasbarrini, "The role of methane in intestinal diseases," American Journal of Gastroenterology, vol. 105, no. 6, pp. 1250-1256, 2010.

[7] S.-Y. Mao, C.-F. Yang, and W.-Y. Zhu, "Phylogenetic analysis of methanogens in the pig feces," Current Microbiology, vol. 62, no. 5, pp. 1386-1389, 2011. 
[8] Y. H. Luo, Y. Su, A. D. G. Wright, L. L. Zhang, H. Smidt, and W. Y. Zhu, "Lean breed Landrace pigs harbor fecal methanogens at higher diversity and density than obese breed Erhualian pigs," Archaea, vol. 2012, Article ID 605289, 9 pages, 2012.

[9] M. C. Collado, M. Cernada, C. Bauerl, M. Vento, and G. PerezMartinez, "Microbial ecology and host-microbiota interactions during early life stages," Gut Microbes, vol. 3, pp. 352-365, 2012.

[10] C. H. F. Hansen, D. S. Nielsen, M. Kverka et al., "Patterns of early gut colonization shape future immune responses of the host," PLoS ONE, vol. 7, no. 3, Article ID e34043, 2012.

[11] J. M. Saavedra and A. M. Dattilo, "Early development of intestinal microbiota: implications for future health," Gastroenterology Clinic of North America, vol. 41, pp. 717-731, 2012.

[12] S. Matamoros, C. Gras-Leguen, F. le Vacon, G. Potel, and M. F. de la Cochetiere, "Development of intestinal microbiota in infants and its impact on health," Trends in Microbiology, vol. 21, pp. 167-173, 2013.

[13] S. Fanaro, R. Chierici, P. Guerrini, and V. Vigi, "Intestinal microflora in early infancy: composition and development," Acta Paediatrica, vol. 91, supplement 441, pp. 48-55, 2003.

[14] C. Palmer, E. M. Bik, D. B. DiGiulio, D. A. Relman, and P. O. Brown, "Development of the human infant intestinal microbiota," PLoS Biology, vol. 5, no. 7, article e177, 2007.

[15] P. Panigrahi, S. Parida, L. Pradhan et al., "Long-term colonization of a Lactobacillus plantarum synbiotic preparation in the neonatal gut," Journal of Pediatric Gastroenterology and Nutrition, vol. 47, no. 1, pp. 45-53, 2008.

[16] C. L. Thompson, B. Wang, and A. J. Holmes, "The immediate environment during postnatal development has long-term impact on gut community structure in pigs," ISME Journal, vol. 2, no. 7, pp. 739-748, 2008.

[17] M. E. Conroy, H. N. Shi, and W. A. Walker, "The long-term health effects of neonatal microbial flora," Current Opinion in Allergy and Clinical Immunology, vol. 9, no. 3, pp. 197-201, 2009.

[18] X. Guo, X. Xia, R. Tang, and K. Wang, "Real-time PCR quantification of the predominant bacterial divisions in the distal gut of Meishan and Landrace pigs," Anaerobe, vol. 14, no. 4, pp. 224-228, 2008.

[19] E. O. Casamayor, R. Massana, S. Benlloch et al., "Changes in archaeal, bacterial and eukaryal assemblages along a salinity gradient by comparison of genetic fingerprinting methods in a multipond solar saltern," Environmental Microbiology, vol. 4, no. 6, pp. 338-348, 2002.

[20] M. J. L. Coolen, E. C. Hopmans, W. I. C. Rijpstra et al., "Evolution of the methane cycle in Ace Lake (Antarctica) during the Holocene: response of methanogens and methanotrophs to environmental change," Organic Geochemistry, vol. 35, no. 10, pp. 1151-1167, 2004.

[21] E. Stackebrandt and B. M. Goebel, "Taxonomic note: a place for DNA-DNA reassociation and 16S rRNA sequence analysis in the present species definition in bacteriology," International Journal of Systematic Bacteriology, vol. 44, no. 4, pp. 846-849, 1994.

[22] S. Wu, Z. Zhu, L. Fu, B. Niu, and W. Li, "WebMGA: a customizable web server for fast metagenomic sequence analysis," $B M C$ Genomics, vol. 12, article 444, 2011.

[23] J. Good, "The population frequencies of species and the estimation of population parameters," Biometrika, vol. 40, pp. 237-264, 1953.

[24] P. D. Schloss, S. L. Westcott, T. Ryabin et al., "Introducing mothur: open-source, platform-independent, communitysupported software for describing and comparing microbial communities," Applied and Environmental Microbiology, vol. 75, no. 23, pp. 7537-7541, 2009.

[25] C. Lozupone and R. Knight, "UniFrac: a new phylogenetic method for comparing microbial communities," Applied and Environmental Microbiology, vol. 71, no. 12, pp. 8228-8235, 2005.

[26] J. D. Thompson, D. G. Higgins, and T. J. Gibson, "CLUSTAL $\mathrm{W}$ : improving the sensitivity of progressive multiple sequence alignment through sequence weighting, position-specific gap penalties and weight matrix choice," Nucleic Acids Research, vol. 22, no. 22, pp. 4673-4680, 1994.

[27] N. Saitou and M. Nei, "The neighbor-joining method: a new method for reconstructing phylogenetic trees," Molecular Biology and Evolution, vol. 4, no. 4, pp. 406-425, 1987.

[28] K. Takai and K. Horikoshi, "Rapid detection and quantification of members of the archaeal community by quantitative PCR using fluorogenic probes," Applied and Environmental Microbiology, vol. 66, no. 11, pp. 5066-5072, 2000.

[29] R. E. Ley, F. Bäckhed, P. Turnbaugh, C. A. Lozupone, R. D. Knight, and J. I. Gordon, "Obesity alters gut microbial ecology," Proceedings of the National Academy of Sciences of the United States of America, vol. 102, no. 31, pp. 11070-11075, 2005.

[30] B. S. Samuel and J. I. Gordon, "A humanized gnotobiotic mouse model of host-archaeal-bacterial mutualism," Proceedings of the National Academy of Sciences of the United States of America, vol. 103, no. 26, pp. 10011-10016, 2006.

[31] S. E. Denman, N. W. Tomkins, and C. S. McSweeney, "Quantitation and diversity analysis of ruminal methanogenic populations in response to the antimethanogenic compound bromochloromethane," FEMS Microbiology Ecology, vol. 62, no. 3, pp. 313-322, 2007.

[32] A.-D. G. Wright, C. H. Auckland, and D. H. Lynn, "Molecular diversity of methanogens in feedlot cattle from Ontario and Prince Edward Island, Canada," Applied and Environmental Microbiology, vol. 73, no. 13, pp. 4206-4210, 2007.

[33] B. Dridi, M. Henry, A. El Khéchine, D. Raoult, and M. Drancourt, "High prevalence of Methanobrevibacter smithii and Methanosphaera stadtmanae detected in the human gut using an improved DNA detection protocol," PLoS ONE, vol. 4, no. 9, Article ID e7063, 2009.

[34] F. Armougom, M. Henry, B. Vialettes, D. Raccah, and D. Raoult, "Monitoring bacterial community of human gut microbiota reveals an increase in Lactobacillus in obese patients and Methanogens in anorexic patients," PLoS ONE, vol. 4, no. 9, Article ID e7125, 2009.

[35] M. Million, M. Maraninchi, M. Henry et al., "Obesity-associated gut microbiota is enriched in Lactobacillus reuteri and depleted in Bifidobacterium animalis and Methanobrevibacter smithii," International Journal of Obesity, vol. 36, pp. 817-825, 2012.

[36] H. H. Wenzl, G. Schimpl, G. Feierl, and G. Steinwender, "Time course of spontaneous bacterial translocation from gastrointestinal tract and its relationship to intestinal microflora in conventionally reared infant rats," Digestive Diseases and Sciences, vol. 46, no. 5, pp. 1120-1126, 2001.

[37] A. K. Benson, S. A. Kelly, R. Legge et al., "Individuality in gut microbiota composition is a complex polygenic trait shaped by multiple environmental and host genetic factors," Proceedings of the National Academy of Sciences of the United States of America, vol. 107, no. 44, pp. 18933-18938, 2010.

[38] C. F. Favier, W. M. de Vos, and A. D. L. Akkermans, "Development of bacterial and bifidobacterial communities in feces of newborn babies," Anaerobe, vol. 9, no. 5, pp. 219-229, 2003. 
[39] P. A. Vaishampayan, J. V. Kuehl, J. L. Froula, J. L. Morgan, H. Ochman, and M. P. Francino, "Comparative metagenomics and population dynamics of the gut microbiota in mother and infant," Genome Biology and Evolution, vol. 2, no. 1, pp. 53-66, 2010.

[40] S. R. Konstantinov, A. A. Awati, B. A. Williams et al., "Postnatal development of the porcine microbiota composition and activities," Environmental Microbiology, vol. 8, no. 7, pp. 11911199, 2006.

[41] T. L. Miller and C. Lin, "Description of Methanobrevibacter gottschalkii sp. nov., Methanobrevibacter thaueri sp. nov., Methanobrevibacter woesei sp. nov. and Methanobrevibacter wolinii sp. nov," International Journal of Systematic and Evolutionary Microbiology, vol. 52, no. 3, pp. 819-822, 2002.

[42] M. G. Dominguez-Bello, E. K. Costello, M. Contreras et al., "Delivery mode shapes the acquisition and structure of the initial microbiota across multiple body habitats in newborns," Proceedings of the National Academy of Sciences of the United States of America, vol. 107, no. 26, pp. 11971-11975, 2010.

[43] M.-M. Grönlund, Ł. Grzeskowiak, E. Isolauri, and S. Salminen, "Influence of mother's intestinal microbiota on gut colonization in the infant," Gut Microbes, vol. 2, no. 4, pp. 227-233, 2011.

[44] Y. Qin, S. Zou, Y. Xu, P. Wang, and X. Xu, "Dynamic analyses of lactose and milk protein in Erhualian Sows and their comparisons with Yorkshire sows," Journal of Anhui Agricultural University, vol. 27, pp. 167-170, 2000 (Chinese). 

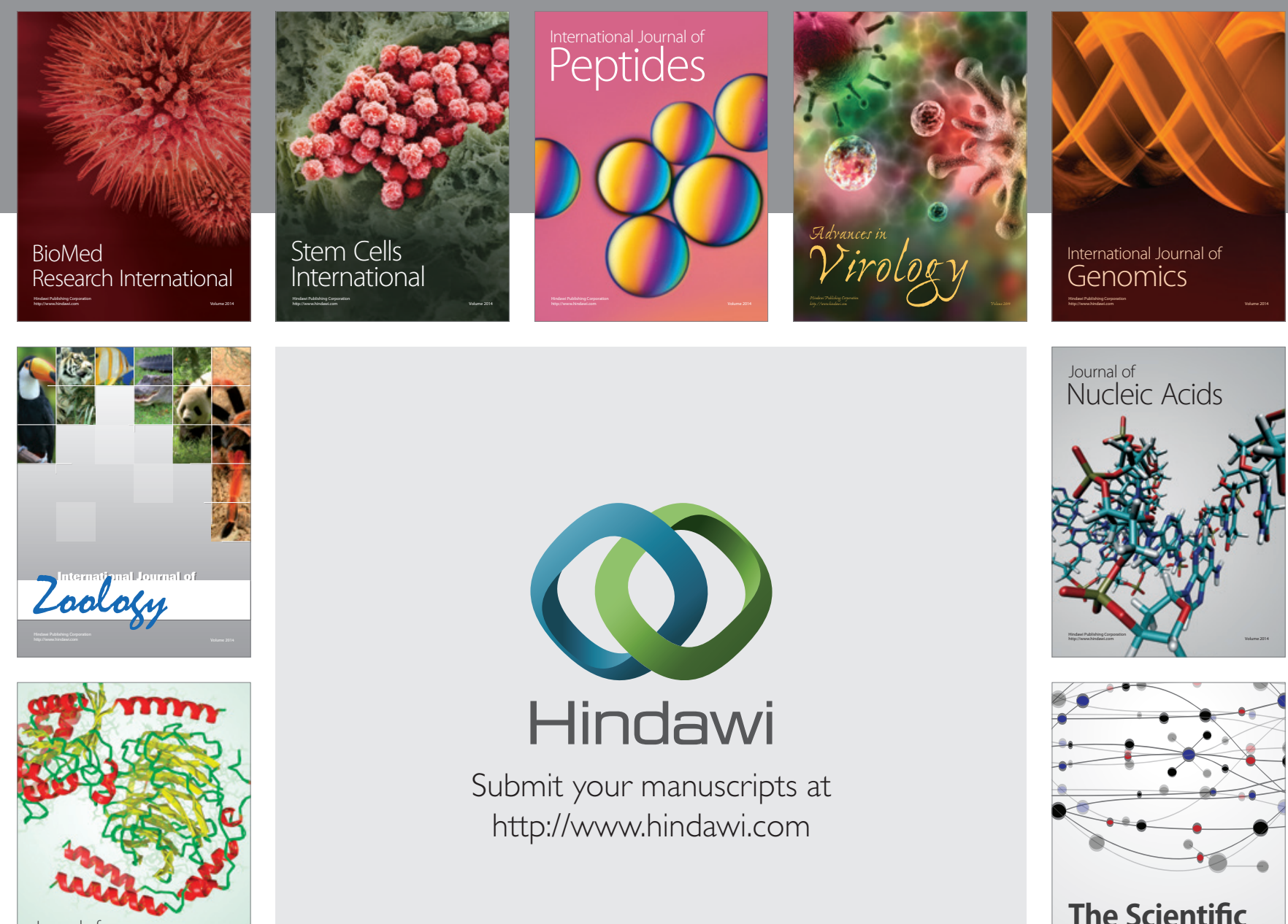

Submit your manuscripts at

http://www.hindawi.com

Journal of
Signal Transduction
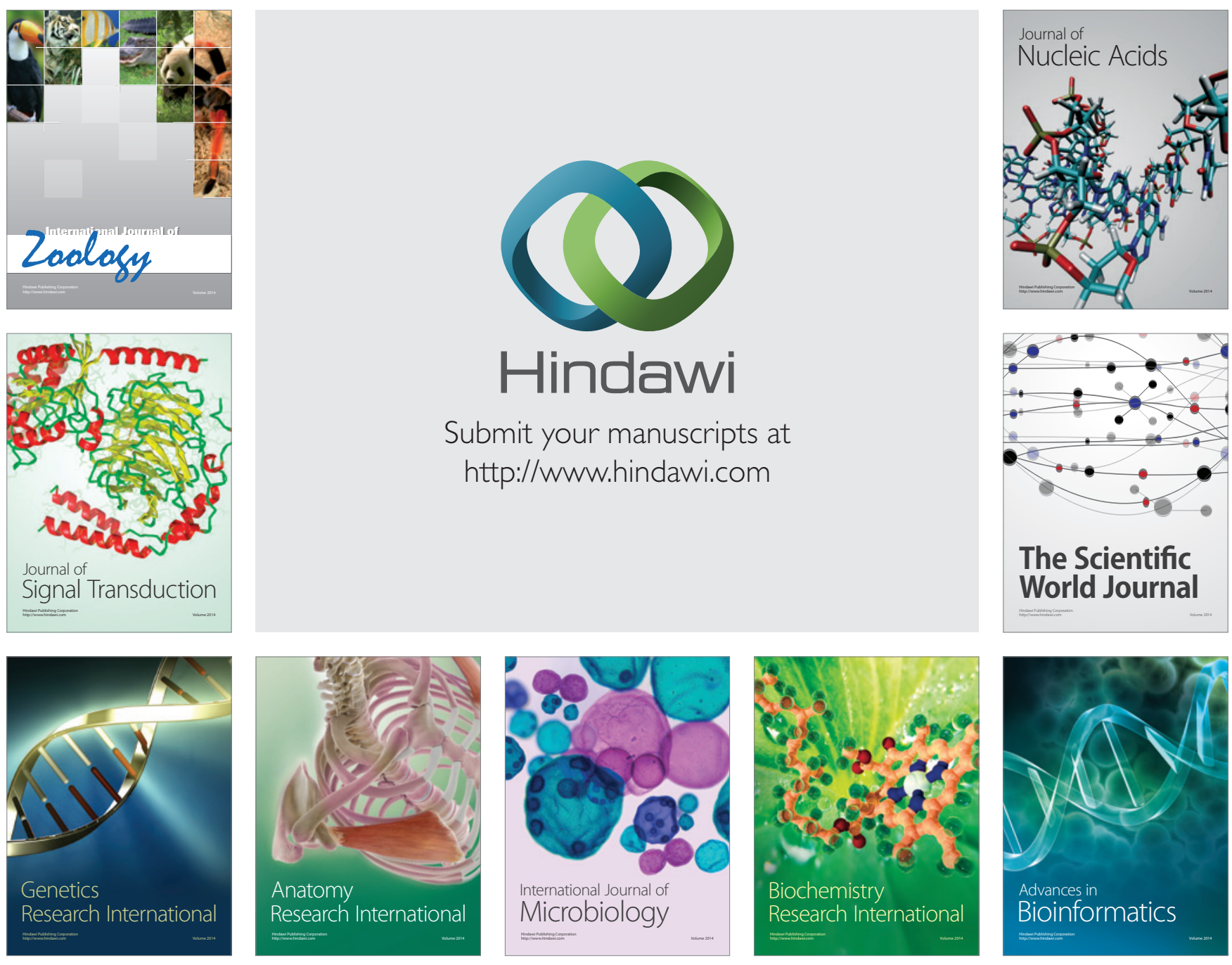

The Scientific World Journal
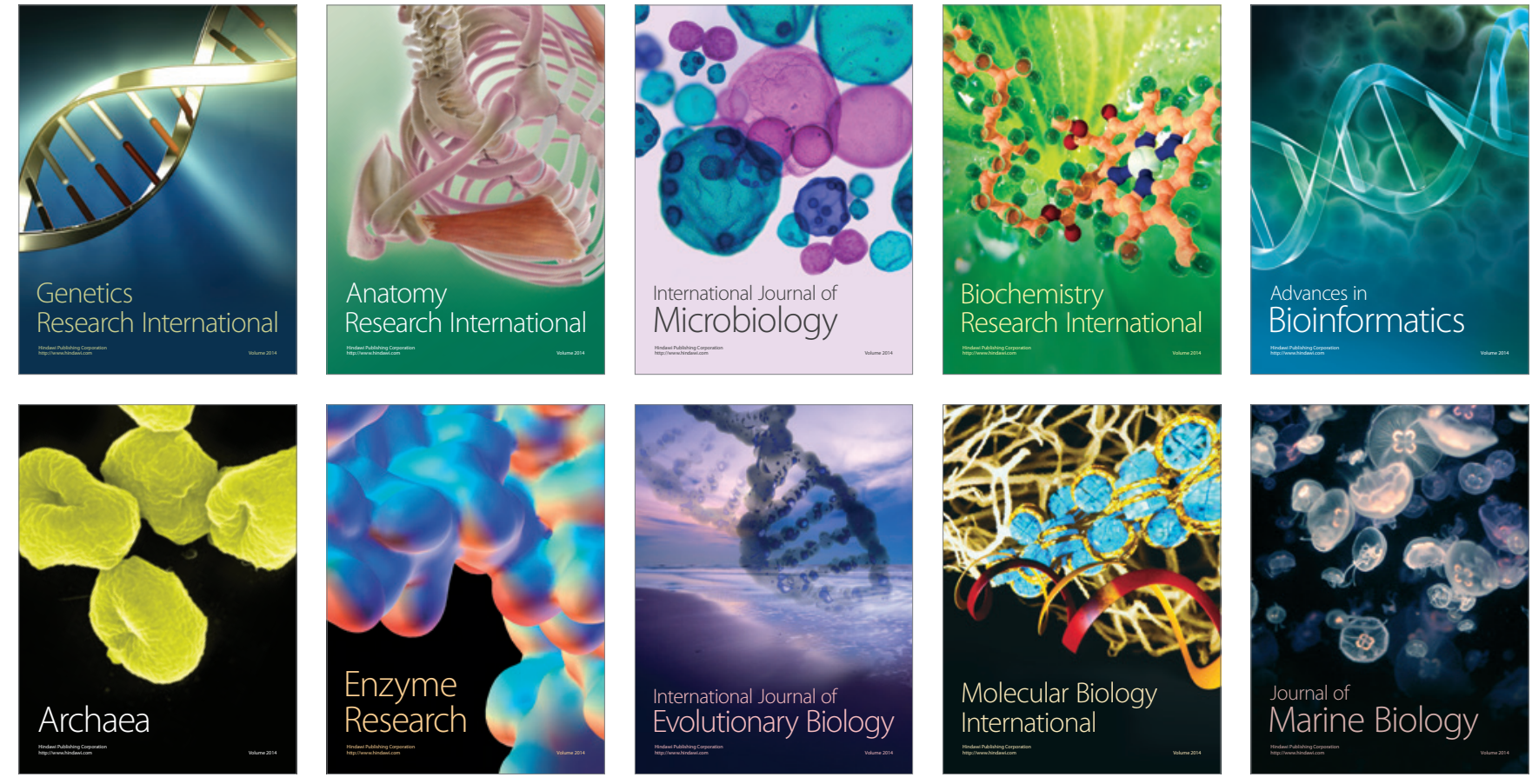